\title{
Transnationalisme des migrants en Europe : une preuve par les faits
}

European migrants' transnationalism: factual evidence

Transnacionalismo de los migrantes en Europa : pruebas al canto

\section{Rosita Fibbi et Gianni D'Amato}

\section{(2) OpenEdition}

Édition électronique

URL : https://journals.openedition.org/remi/4452

DOI : $10.4000 /$ remi.4452

ISSN : $1777-5418$

Éditeur

Université de Poitiers

Édition imprimée

Date de publication : 1 novembre 2008

Pagination : 7-22

ISBN : 978-2-911627-49-0

ISSN : 0765-0752

Référence électronique

Rosita Fibbi et Gianni D'Amato, «Transnationalisme des migrants en Europe : une preuve par les

faits », Revue européenne des migrations internationales [En ligne], vol. 24 - n² | 2008, mis en ligne le 16 décembre 2008, consulté le 14 avril 2022. URL : http://journals.openedition.org/remi/4452 ; DOI :

https://doi.org/10.4000/remi.4452 


\title{
Transnationalisme des migrants en Europe : une preuve par les faits
}

\author{
Rosita FIBBI* et Gianni D'AMATO**
}

Depuis le début des années 1990, la littérature anglo-américaine en sciences sociales sur le transnationalisme n'a cessé de croître. Par transnationalisme, on entend une construction de champs sociaux dans laquelle les migrants créent un lien — imaginaire ou réel — entre leur pays d'origine et leur société d'accueil (Glick Schiller et al., 1992 ; Portes, 1997). Dans ces espaces transnationaux, les migrants mettent en place des relations sociales et économiques, des activités et des identités politiques qui transcendent les frontières classiques et bénéficient de processus économiques globaux à l'intérieur d'un monde divisé en États-Nations. Le transnationalisme représente un déplacement des identités, notamment nationales, et des revendications politiques et sociales au-delà des appartenances territoriales habituelles fondées sur l'échelle des États-Nations. Cette perspective de recherche apparaît incontournable dans un souci de dépassement du nationalisme méthodologique (Wimmer et Glick Schiller, 2002). Ainsi l'approche du transnationalisme a pu se positionner, dans le champ de la migration, comme une sorte de troisième voie entre l'option de l'assimilation et celle du retour, entre une perspective où l'action individuelle est surdéterminée par les contraintes macro-sociales (voir Portes, 1999) et une perspective qui conçoit celle-ci comme le résultat de la volonté et des préférences des acteurs sociaux (voir Kivisto, 2001).

Si la littérature théorique sur le transnationalisme est bien développée, la base empirique est restée plutôt limitée : il existe peu de descriptions systématiques des pratiques transnationales des migrants. Cela est particulièrement vrai en Europe continentale où la recherche sur ce phénomène est faiblement représentée (Rogers, 2000 ; Waterbury, 2008) ${ }^{1}$. L'Europe, avec ses nombreux États-nations et ses flux

* Forum suisse pour l'étude des migrations et de la population, Université de Neuchâtel, Rosita.fibbi@unine.ch

** Forum suisse pour l'étude des migrations et de la population, Université de Neuchâtel, Gianni.damato@unine.ch

1 Pour une documentation des débats les plus récents, voir les papiers présentés à l'occasion de la conférence « Diaspora and Transnationalism : Conceptual, Theoretical and Methodological Challenges », Institut Universitaire Européen, Florence, 10-11 avril 2008. 
migratoires importants, connaît-elle des formes de transnationalisme comme cela semble être le cas dans le monde anglo-américain ? Comparant des études transnationales aux États-Unis et en Europe, Østergaard-Nielsen (2003) met l'accent sur le fait qu'en Europe, l'attention est focalisée sur les pays de réception ainsi que sur les idéologies et la politique, alors qu'aux États-Unis, l'accent est mis sur les pays de départ et sur le transnationalisme de base (« grassroot transnationalism »).

Le projet de recherche qui est à l'origine de ce dossier ${ }^{2}$ a cherché à répondre à ces questions en se centrant sur le transnationalisme de base. L'objectif poursuivi était de sonder la portée des activités transnationales par-delà les observations non systématiques actuellement existantes et de réaliser une évaluation comparative des activités transnationales de différents groupes immigrés dans divers pays. Sur la base de matériaux empiriques documentant les activités transnationales de ces groupes, le projet vise à développer des hypothèses explicatives sur les facteurs politiques, culturels et économiques qui conditionnent la création et la reproduction des activités transnationales.

En conséquence, ce ne sont pas les communautés qui sont au centre de l'étude, mais les pratiques transnationales. Les pratiques transnationales sont les activités déployées par les migrants impliquant au moins deux pays, incluant des activités entre le pays de résidence et le pays d'origine et/ou des ancêtres, ainsi que les activités menées dans des pays tiers, quel que soit le degré d'implication des co-ethniques. $\mathrm{Ne}$ sont retenues dans l'étude que les activités qualifiées de transnationales par Portes dans sa typologie fondée sur les acteurs (Portes, 2001), à savoir celles « initiées et soutenues par les acteurs non institutionnels, par leurs groupes organisés ou leurs réseaux individuels à travers les frontières ». Sont ainsi exclus les activités et programmes des États-Nations qualifiés d'internationaux et les activités des institutions de large échelle telles que les corporations ou les Églises, qualifiées des multinationales. Ainsi, les dimensions collectives des liens transfrontaliers, leur ouverture sur l'espace public, voire les formes de consolidation institutionnelles «par le bas » des pratiques individuelles sont au centre de ce projet.

Trois sphères sociales, la sphère économique, la sphère socioculturelle et la sphère politique font l'objet de l'analyse : en dépit de la difficulté d'un tel découpage de la réalité et de la perméabilité de ces sphères, le souhait était de se donner les moyens de voir apparaître l'ensemble des activités, et non seulement les activités politiques, qui ont dominé jusqu'ici les études empiriques sur le transnationalisme, notamment en Europe. Cette recherche reflète le souci de s'orienter vers la description (Berthomière et Hily, 2006 : 72) et vise à montrer la réalité sociale des activités transnationales sur une base comparative, tout en observant les interférences et les interactions des pays d'immigration et d'origine avec les communautés étudiées.

2 Le réseau établi pour mener à bien cette étude rassemble des chercheurs européens travaillant sur les migrations, la circulation migratoire et les politiques d'intégration réunis dans le cadre du Network of Excellence IMISCOE du $6^{\text {ème }}$ Programme Cadre européen (http://www.imiscoe.org/). 
Dans ce cadre, la spécificité de cette étude réside dans sa méthodologie multicomparative. Les études sur la migration sont articulées autour de trois approches comparatives principales : dans l'approche convergente, l'étude de différents groupes dans un seul pays privilégie la perspective du pays d'immigration et tend à imputer les différences aux caractéristiques des groupes immigrés ${ }^{3}$; dans l'approche divergente, l'étude d'un même groupe immigré dans différents pays dirige le regard vers la variabilité des conditions des pays d'accueil ; la troisième approche vise la comparaison d'un groupe migrant avant sa migration dans le pays d'envoi et après sa migration dans le pays de résidence (Green, 2002).

Le projet a cherché à reproduire ces trois situations de comparaison. Dès lors les études présentées dans ce numéro de la REMI n'ont pas toutes la même structure Trois des études constituent des contributions articulées autour d'une approche divergente : les Turcs sont analysés dans trois pays différents d'installation, les PaysBas, l'Allemagne et la Suisse. Les cas des Marocains en Belgique, en France et en Italie sont appréhendés dans chaque article en parallèle avec un autre groupe immigré également établi dans le pays. Quatre autres contributions sont conçues comme des comparaisons convergentes entre deux groupes différents installés dans un même pays d'immigration selon une approche plus habituelle dans les études sur les migrations ; ainsi les Marocains sont comparés aux Chinois en Belgique, aux Sénégalais en France et aux Roumains en Italie. Finalement, dans le cas des Portugais ainsi que des Roumains, nous avons tenté d'explorer les pratiques transnationales dans la perspective du pays d'origine et dans celle du pays d'accueil.

\section{LES RÉSULTATS EMPIRIQUES}

Remettant en discussion la focalisation exclusive sur la relation entre immigrés et pays d'accueil caractéristique des perspectives assimilationnistes, l'approche du transnationalisme prend en compte également les liens et les activités déployés par les migrants avec leur pays d'origine et les membres de leurs groupes d'origine migrés vers d'autres destinations. Dans cette optique, l'on pourrait s'attendre à ce que les flux migratoires les plus fournis et les plus récents soient caractérisés par une grande richesse des liens transnationaux, justement en raison de la pertinence encore très forte pour les émigrés des enjeux liés au pays d'origine.

Ainsi est-il surprenant de constater la relative faiblesse des activités transfrontalières chez les groupes qui connaissent une immigration conséquente et récente.

3 Il s'agit de groupes de même nationalité d'origine, composites cependant d'un point de vue ethnique, social ou du genre. Il convient en effet de contourner l'écueil implicite de la notion de «communauté », à savoir le présupposé qu'il s'agit d'un groupe délimité, fermé et homogène. Nous avons dès lors choisi une unité d'analyse ouverte, qui évite l'essentialisation et la généralisation à l'ensemble du groupe national. Cependant, même l'étude des pratiques et des conditions de leur développement comporte comme instrument d'analyse un groupe défini sociologiquement, car ce n'est qu'ainsi que se laisse appréhender l' «agency » qui structure les groupes (Ragazzi, $2008: 13$ ). 
Le cas des primo-migrants Portugais en Suisse et des Roumains et des Marocains en Italie nous interpellent, justement parce qu'ils sont à première vue contre-intuitifs. C'est d'autant plus étonnant que ces flux sont largement habités par la perspective du retour dans le pays d'origine.

Toutefois, ce trait n'est guère une spécificité de tous les groupes émigrés en provenance de ces pays : les Marocains de France et de Belgique, en effet, déploient une intense activité transnationale sur les plans économique, culturel et politique. Ils se rapprochent ainsi du cas des Turcs, le groupe migrant le plus important numériquement en Europe, installé dans divers pays d'immigration. Actifs à différents niveaux, les Turcs constituent désormais l'exemple paradigmatique des transmigrants en Europe. À ce titre, ils ont fait l'objet de nombreuses études, portant tout particulièrement sur leur intense activité politique en direction du pays d'origine (Bauböck, 1994b ; Faist, 2000 ; Koopmans et Statham, 2001 ; Lucassen, 2002 ; Østergaard-Nielsen, 2003), qui toutefois ont plutôt laissé dans l'ombre les intenses relations tissées entre les groupes d'origine turque établis dans divers pays européens.

Finalement, les autres groupes étudiés dans ce projet se situent dans des positions intermédiaires en ce qui concerne l'intensité de leurs activités transnationales mais surtout le spectre des activités déployées : les Capverdiens du Portugal, les Chinois de Belgique et les Sénégalais de France se distinguent par le fait qu'ils investissent de manière privilégiée les champs économique et socioculturel alors que l'activité politique, sans être inexistante, n'est de loin pas prioritaire.

Le «faible transnationalisme » observé à propos des Portugais en Suisse ou des Marocains et Roumains en Italie n'est pas une allusion au fait que - comme il est désormais admis dans la recherche sur le transnationalisme - le nombre de personnes effectivement engagées dans des activités de ce type demeure limité (Levitt, 2002 : 19). Cette expression souligne plutôt la faible ouverture de ces activités sur l'espace public. Certes, nombreux sont les individus dans chaque groupe qui envoient régulièrement un soutien financier à leurs familles, investissent leur épargne pour préparer leur retour, soignent les relations avec la parenté et les amis de leur premier lieu de socialisation par le biais de contacts téléphoniques ou épistolaires, ou encore suivent l'actualité politique et/ou sportive via les journaux ou la télévision. Ces activités n'acquièrent cependant pas de relevance au-delà du cercle privé, familial ou parental et ne sont ainsi pas prises en considération dans ce projet.

\section{ÉMERGENCE DE PRATIQUES TRANSNATIONALES COLLEC- TIVES : UN EFFET DE SEUIL}

Les liens transfrontaliers devraient être les plus développés chez les groupes qui connaissent une immigration récente et numériquement importante, si l'on suit la logique de l'assimilation (Alba et Nee 2003 ; Waldinger 2006). D'ailleurs, comme attestent la plupart des études sur les pratiques transnationales, c'est la première génération qui s'engage le plus dans le maintien de liens par-dessus les frontières. Ainsi, la faiblesse des activités transfrontalières mise en lumière par les études de cas 
sur les primo-migrants portugais en Suisse, ainsi que roumains et marocains en Italie, paraît paradoxale. Or, ce paradoxe n'est un qu'à condition de croire que la constitution de réseaux transfrontaliers est la conséquence automatique du déplacement : en effet, si l'identification au pays/à la région d'origine est vive chez les primo-migrants, elle ne se concrétise en activités transfrontalières que lorsque certaines conditions sont réunies.

Les Marocains et les Roumains en Italie ont un statut juridique relativement incertain dans le pays d'immigration et sont souvent en situation précaire sur le plan économique : arrivés récemment en Italie, ils sont absorbés par la tâche de consolider leur présence, voire leur survie. Ainsi la menace de marginalisation et de paupérisation semble inhiber les possibilités de développer des activités transnationales.

L'argument de la précarité contribue à expliquer la différence entre les Marocains en Italie et ceux établis en France et en Belgique : les premiers font partie d'un flux migratoire encore relativement récent alors que la présence des Marocains immigrés dans les deux pays francophones s'est consolidée au cours de plusieurs décennies. Contrairement à l'hypothèse qui suggère que le développement des activités transnationales est une réponse à l'incertitude de l'insertion des immigrés dans leur nouveau pays (Portes, 1997), il apparaît plutôt que trop de précarité s'avère paralysante, notamment lorsqu'elle est associée à des maigres ressources personnelles et sociales des membres du groupe. C'est aussi la conclusion à laquelle parvient Dahinden dans son étude des réseaux sociaux individuels des Albanais en Suisse : "The 'missing' transnationalism could at least partially be explained by the disadvantaged and underprivileged position of the Albanians migrants and by their limited personal resources, particularly in terms of cultural capital » (Dahinden, $2005: 13)$.

Un deuxième argument paraît pertinent, celui de la diversification interne des groupes immigrés, en termes de type de migration ou de capital socio-culturel : la migration marocaine en France et en Belgique, tout en étant largement le fait de travailleurs et de leurs familles, présente une composante politique qui est largement absente parmi les Marocains d'Italie. Portes le relevait déjà : «Where migration is a more individualized process, grounded on personal and family decisions, transnational activities are more selective and, at times, exceptional, lacking the normative component attached to them among participants in a political diaspora » (Portes, 1999 : 464). Finalement, on pourrait imaginer que les Marocains d'Italie diffèrent de part leur provenance régionale de ceux émigrés vers la France, une piste d'étude intéressante, toutefois encore largement inexplorée (Eve dans ce volume).

L'argument de la précarité du statut et de la condition sociale est en revanche de peu de secours pour rendre compte de la faiblesse des activités transnationales dans le cas d'une immigration aussi soutenue que celle des Portugais en Suisse. Présents depuis 30 ans, ils sont toujours nombreux à être engagés dans une migration «à terme », impliquant souvent le retour définitif, rendu possible justement par l'accès privilégié aux droits de résidence dérivant de l'appartenance du pays à l'Union européenne. Brettel (1986), qui a étudié dans les années 1980 les Portugais établis à Paris, 
observe également une forte orientation au retour ainsi qu'une absence d'associations volontaires ${ }^{4}$. Dans ce cas donc, c'est le deuxième argument, celui de l'absence de diversification interne au groupe qui est, vraisemblablement, à l'origine de l'absence relative d'activités transnationales ayant une dimension collective.

\section{PRATIQUES DE TRANSNATIONALISME POLITIQUE}

Les diverses études présentées dans ce dossier montrent que les immigrés turcs, marocains et sénégalais sont engagés dans des pratiques transnationales de type politique dans leurs pays de résidence respectifs. La contribution néerlandaise sur les Turcs explore cette dimension prioritairement, alors que les articles sur les Turcs en Allemagne et en Suisse, les Marocains en Belgique, ainsi que les Sénégalais et les Marocains en France montrent que cette activité politique va largement de pair avec une forte présence transnationale dans les sphères culturelle et économique également. Ainsi la focalisation sur cet aspect de leur expérience transnationale offre une vision approfondie qui toutefois n'épuise pas la richesse et la diversité des activités observables (Amelina et Faist dans ce volume). Certes, la mobilisation politique transnationale est la forme d'action collective la plus visible et aussi celle qui interfère davantage que toute autre avec le cloisonnement de l'espace social et politique en États-nations. Elle représente en effet le défi le plus élevé à la conception traditionnelle des États-nations, qui fonctionnent comme des juridictions légales exclusives. Dès lors, il n'est pas surprenant qu'elle ait souvent attiré l'attention des chercheurs depuis l'émergence de ce champ de réflexion.

L'élément crucial, pour notre analyse comparative des cas de mobilisation politique transnationale des migrants, est constitué par les contextes politiques qui "provide incentives for people to undertake collective action by affecting their expectations for success or failure. » (Tarrow, 1994 : 85). Koopmans et Statham (2001) conçoivent ainsi la structure des opportunités politiques («political opportunity structure » ou POS) comme la disponibilité et l'attractivité relative d'options différentes pour l'action collective dans la perspective des groupes en opposition. Ils identifient trois facteurs déterminant cette mobilisation : (1) l'influence du pays d'accueil par le biais d'une POS ouverte, notamment par le droit de vote local ; (2) l'influence du pays d'origine dans le but de retenir l'allégeance (notamment par le droit de vote dans le pays d'origine); (3) l'identité collective des migrants, telle que façonnée par l'appartenance à un même groupe ethnique, une même communauté religieuse, une même idéologie politique. Koopmans et Statham expliquent l'existence d'activités transnationales par une inclusion politique insuffisante des groupes immigrés dans le pays d'accueil qui, dès lors, adressent leurs revendications au pays d'origine (constraining conditions) ; à l'opposé, Faist (2000) entrevoit les raisons de l'activation politique dans les grandes opportunités fournies aux migrants par le cadre démocratique des pays d'immigration (enabling conditions).

4 En revanche, elle observe une forte activité transnationale chez les Portugais du Canada. 
Tous les groupes étudiés vivent dans des pays démocratiques qui assurent aux migrants un spectre large de droits civils et sociaux; de plus, par le biais de la naturalisation, les droits politiques sont accessibles à une partie non négligeable des membres des migrations les plus anciennes. De plus, l'opportunité de plus en plus fréquente pour les migrants de combiner des statuts et affiliations à la fois externes et internes grâce à la double citoyenneté (Bauböck, 2003 : 701) constitue le levier d'un potentiel engagement politique tant à l'égard de la société d'immigration que de celle d'origine. À cela s'ajoute le droit de vote depuis l'étranger garanti par certains pays d'origine à leurs ressortissants expatriés.

Cette opportunité politique offerte par le pays d'origine ne semble pas avoir l'impact sur la mobilisation des immigrés qu'on aurait pu escompter. Les Capverdiens au Portugal et les Roumains en Italie, qui pourtant jouissent de ce droit de vote, ne se distinguent guère par leurs activités politiques transnationales. Il en va de même pour les Portugais en Suisse, moins actifs politiquement que les Turcs qui ne bénéficient pas du droit de vote depuis l'étranger. Les Italiens aussi, qui ont obtenu le droit de vote à l'étranger en 2001, étaient plus actifs politiquement en Suisse dans les années 1970 et 1980 qu'après l'obtention de ce droit (Fibbi, 1997).

Le comportement «transnational » fort différent des deux groupes immigrés en Suisse, les Turcs et les Portugais, éclaire la nécessité d'articuler entre elles les deux explications classiques de la mobilisation sociopolitique des immigrés, opérant avec le concept de structure des opportunités politiques. La comparaison de ces deux cas confirme plutôt l'hypothèse des «enabling conditions » qui toutefois nécessite une spécification ultérieure : ce ne sont pas les opportunités offertes par le pays d'immigration en tant que telles qui sont déterminantes pour la mobilisation mais plutôt le décalage entre ces opportunités - le plus souvent élargies — dans le pays d'immigration et celles — plutôt restreintes — du pays d'origine (D'Amato et al., 1997). Ce n'est que là où ce contraste est fort que les conditions sont réunies pour une mobilisation. On peut citer à titre d'exemple le cas des Portugais en Suisse dans les années 1960 et début 1970 : tant que cette différence entre pays d'origine et de résidence existait pour eux, de nombreux intellectuels portugais en Suisse déployaient une activité politique transnationale (Pache, 1990) disparue une fois la démocratie rétablie au pays d'origine. Au-delà du cas suisse, c'est ce contraste qui est susceptible d'expliquer la mobilisation politique des Sénégalais, mais aussi des Turcs et des Marocains, du moins à partir des pays où ces groupes ont acquis un statut social, économique et politique consolidé.

Cette différence structurelle est une condition d'autant plus indispensable quand la mobilisation politique des immigrés canalise des ressources sociales du groupe accumulées dans le pays de résidence et est orientée vers le pays d'origine (homeland directed transnationalism) (Østergaard-Nielsen, 2003). C'est le type de mobilisation qui prévaut chez les Marocains et les Sénégalais étudiés dans ce projet alors que dans le cas turc, on trouve, outre ce premier type, des revendications articulées dans le pays de résidence mais concernant le pays d'origine (transplanted homeland politics) (Nell dans ce volume). 
En revanche, l'indéniable décalage entre droits politiques dans le pays d'immigration et droits politiques dans le pays d'origine ne semble guère stimuler une mobilisation politique des Chinois de Belgique, pourtant souvent naturalisés et bien dotés en ressources économiques et sociales. Dès lors, c'est que d'autres conditions - par exemple la diversification interne du groupe et l'élaboration de formes d'identité collective — ne sont pas réunies dans le cas des Chinois en Belgique ${ }^{5}$. Dans ce contexte, leurs ressources culturelles et symboliques sont davantage canalisées vers les activités économiques et culturelles (Bousetta et Martiniello dans ce volume).

\section{PRATIQUES DE TRANSNATIONALISME ÉCONOMIQUE ET CULTUREL}

Entre ces deux extrêmes que sont le cantonnement dans des activités économiques individuelles destinées à sa propre famille d'une part, et une vive activité politique orientée tant vers le pays d'origine que vers celui de résidence d'autre part, les recherches conduites dans le cadre de notre projet identifient des situations intermédiaires, où les groupes immigrés ne développent pas une action de revendication, au plan politique mais se profilent comme acteurs sociaux via une activité économique et culturelle à l'échelle transnationale.

Contrairement au transnationalisme politique, les activités transnationales économiques, sociales et culturelles ne touchent guère au lien entre migration et Étatnation (Bauböck, 2003). Ainsi, ce n'est pas un hasard si la deuxième génération, notamment des Turcs en Suisse et des Marocains en Belgique, s'y implique tout particulièrement en trouvant dans ces activités une forme d'expression de leur loyauté au pays d'origine qui ne remet pas en cause leur condition de résidents installés à demeure dans le pays d'immigration ${ }^{6}$. L'implication de la deuxième génération dans ces pratiques ressemble ainsi davantage à des formes de mobilisation de la société civile, dans une perspective plutôt translocale visant à soutenir des localités spécifiques dans le pays d'origine (Levitt, 2007 : 78).

Dans ce cadre, si le contact avec les autorités locales des pays ou régions d'origine demeure nécessaire sur le plan pratique voire logistique, il apparaît à la portée des groupements de migrants. Les régions et les communes sont des «polities » au spectre d'activité limité qui ne contrôlent pas la politique d'immigration et ne sont pas engagées dans un processus de construction nationale. Ces collectivités peuvent ainsi se montrer plus réceptives aux intérêts translocaux des migrants que les institutions nationales. Ainsi observe-t-on dans ces activités une tentative réussie d'autonomisation des individus par rapport au fait national (Fibbi et Meyer, 2002) : elles se

5 Il manque, dans notre approche par les activités transnationales, les éléments permettant d'explorer davantage cette piste.

6 Ces associations diffèrent des «Home Town Associations » qui ont fleuri parmi les immigrés latino-américains aux États-Unis, où l'on trouve davantage la première génération de migrants. 
concrétisent en effet avant tout par le biais d'ONG qui semblent jouir d'une grande autonomie dans la gestion des projets ${ }^{7}$. Il est intéressant de remarquer que dans ce domaine, les deuxièmes générations s'efforcent de créer des canaux nouveaux comme des fondations pour l'amélioration des conditions de vie dans le village d'origine, se détachant des formes plus politisées de participation sociopolitique prédominantes auprès de la première génération (Kaya et Baglioni dans ce volume). La réappropriation de l'initiative et de l'action par les migrants paraît ainsi être une des conditions pour négocier le changement du lien entre migrants de deuxième génération et pays d'origine : ainsi les jeunes se détachent des préoccupations coutumières et religieuses qui, au contraire, caractérisent la relation au village d'origine de leurs aînés (Lacroix et al. dans ce volume $)^{8}$.

La plupart des activités économiques transnationales recensées sont plus amples que le soutien au développement de la région d'origine des migrants. Un des facteurs susceptibles d'expliquer l'entreprenariat immigré et partant l'activation économique transnationale est l'hostilité à laquelle le groupe immigré est confronté dans la nouvelle société (Levitt, 2002 : 18). Les hypothèses du désavantage ethnique qui confine les migrants aux secteurs marginaux d'activité économique (Logan et al., 2003) voire celle de la mobilité bloquée qui frappe les plus qualifiés parmi les migrants (Raijman et Tienda, 2000) trouvent bien confirmation dans l'évidence empirique réunie dans les contributions sur les Turcs en Suisse et en Allemagne ou encore sur les Marocains en Belgique.

Toutefois certaines activités recensées dans les études présentées dans ce numéro vont bien au-delà de la seule réaction à l'hostilité de leur environnement (par exemple le commerce sédentaire des Sénégalais) ou alors elle se meuvent dans des logiques complètement différentes (par exemple les investissements des Chinois), engagées qu'elles sont dans des entreprises de reproduction identitaire s'adressant au marché intra-communautaire (Ma Mung, 1992) mais aussi à la réalisation d'un projet axé sur la circulation migratoire. Les théories explicatives des activités économiques transnationales paraissent insuffisantes à cueillir la grande variabilité des situations observées.

Les nombreuses activités culturelles nourrissent l'identification des individus à la culture d'origine et visent en même temps l'élaboration d'une identité collective positive dans le pays d'immigration. Les immigrés doivent en tant qu'individus se mettre soi-même en relation avec la société : " se mettre en relation » veut dire

7 Ces activités de la société civile sont parfois menées sous l'égide des autorités du pays d'origine ; aux yeux des personnes impliquées cela n'affecte pas leur autonomie mais concourt plutôt à l'efficacité de leur action. La question de l'instrumentalisation des diasporas (Fibbi et Meyer, 2002) reste posée, mais elle est moins saillante que dans les activités politiques proprement dites.

8 D'autres exemples, similaires à celui-ci, de transformation des canaux exprimant le rapport au pays d'origine chez les nouvelles générations ont été décrits pour les Chiliens (Bolzman, 2002) ou les Italiens en Suisse (La Barba et Cattacin, 2007). 
appréhender les autres dans leur altérité et prendre position à leur égard. Dans les sociétés complexes et différenciées, ceci implique une confrontation constante avec tous les possibles projets identitaires, qui résonnent éventuellement comme une remise en question de soi et exigent dès lors soit une confirmation de son propre projet soit sa remise en question. En rendant possible une interaction entre des cultures d'origine des immigrants et les cultures du lieu d'installation, la musique (et la langue, à une échelle plus réduite) permet de comprendre la logique du transnationalisme comme un territoire unique d'action sociale où les immigrants ne sont plus déracinés, mais, au contraire, ils se meuvent librement de côté et d'autre, à travers de différentes cultures et systèmes sociaux (Brettell et Hollifield, 2000) (Marques et Gois dans ce volume).

\section{LES QUESTIONS OUVERTES}

Dans l'impossibilité de traiter ici l'ensemble des questions soulevées dans notre projet initial (D'Amato, 2004), nous en avons retenues trois qui apparaissent comme cruciales pour le développement de nouvelles pistes de recherche dans ce champ ; elles s'articulent autour du caractère « durable » des activités transnationales, des acteurs engagés dans ces activités et finalement du rapport entre engagement transnational et projet migratoire.

\section{Quelle pérennité?}

La dimension diachronique est entrée assez rapidement dans le débat théorique suscité par l'apparition de ce concept, la question étant de savoir si ces intenses activités tissées par-dessus les frontières constituaient un novum historique. Les études des années 2000 (Pries, 2001 ; Vertovec, 2003) tiennent compte des apports des historiens (Lucassen et Lucassen, 1997) et ne revendiquent guère le caractère d'un phénomène inédit pour le transnationalisme actuel. Cela ne veut pas dire pour autant que le transnationalisme d'aujourd'hui ne présente pas de traits spécifiques en comparaison des expériences historiques antérieures, qui tiennent notamment aux formes institutionnelles de l'activité politique et des conceptions de l'appartenance. Dans le nouveau contexte de la globalisation, les liens transnationaux ne sont plus stigmatisés ${ }^{9}$. De plus, l'extension du droit de vote à l'échelle locale pour les «denizens ${ }^{10}$, du droit de vote à l'étranger pour les expatriés, et de la double nationalité représente une nouveauté absolue historiquement, entre autres à cause du nombre élevé de personnes qui en jouissent; elle n'est plus regardée a priori comme une menace pour les États-nations.

9 Dans les années 1960, par exemple, la Convention de Strasbourg (6 mai 1963) sur la réduction des cas de pluralité de nationalité prévoit que l'on ne peut posséder qu'une seule nationalité.

10 Hammar (1990) qualifie de «denizens » cette population étrangère qui réside dans le pays d'immigration depuis longtemps et a développé des formes partielles de participation dans la société de ce pays, sans pour autant en posséder la citoyenneté formelle. 
Notre dispositif d'observation synchronique est inadéquat pour entrer dans ce questionnement historique, qui par ailleurs est aujourd'hui largement résolu. En revanche il permet de thématiser un autre aspect de la dimension diachronique en traitant de la pérennité des activités transnationales des migrants, par le biais de la mobilisation au fil des générations. Bien que le concept de transnationalisme ait été forgé en parallèle avec l'analyse des liens entre les primo-migrants et leur pays d'origine (Portes et al., 1999), le thème de la présence de la deuxième génération, ou de son absence, a acquis assez rapidement un grand relief.

La littérature démontre largement que la durée prolongée du séjour et la succession des générations contribuent à l'affaiblissement des liens transnationaux, notamment à cause de la perte des compétences linguistiques soulignée par Fishman (1964) ou par Gordon (1964) dans sa théorie de l' «Assimilation in American life ». Ces travaux trouvent une confirmation dans des recherches récentes menées aux ÉtatsUnis (Rumbaut, 2002) ainsi qu'en Allemagne ou en Suisse (Nauck et al., 1997 ; Wimmer, 2004), où l'on fait état, par exemple, du relâchement des liens ethniques parmi les Turcs.

Et pourtant cette règle générale souffre d'exceptions. En discutant le cas des gens de couleur aux États-Unis, Fouron et Glick-Schiller suggèrent que l'accueil hostile dont ils ont été la cible soutient la motivation des deuxièmes générations à rester en contact avec la société d'origine de leurs parents, un contexte dans lequel leur condition n'était pas minoritaire et leur statut social les mettait à l'abri des injures liées à une condition raciale stigmatisée (Fouron et Glick Schiller, 2002). C'est dans cette optique que l'on peut comprendre les exemples d'implication des jeunes d'origine marocaine ou turque dans des activités destinées à leur pays d'origine, qui prolongent et actualisent l'engagement « existentiel », le commitment de leurs parents.

Certes, cette permanence ne peut être une continuité à l'identique, ne serait-ce que parce que l'environnement social est par ailleurs en mutation. La permanence implique un changement de forme d'expression de cette implication; elle peut faire appel à des ressources sociales et culturelles des descendants de migrants autres que celles dont disposaient leurs parents primo-migrants. Le transnationalisme politique s'estompe au fil des générations au profit d'activités de type économique et culturel, comme le démontrent les exemples des jeunes, Turcs, Sénégalais ou Marocains. Les jeunes construisent par exemple des réseaux de soutien au développement, dans lesquelles leurs expertises techniques et sociales ainsi que leur socialisation politique aux structures du pays de résidence sont davantage mises à contribution.

Un autre exemple de permanence du lien dans le changement des formes d'expression est celui des activités culturelles ; c'est le terrain privilégié d'expression du lien transnational par les deuxièmes générations qui vivent la déterritorialisation du fait culturel (Cohen, 1997). Les musiciens, les écrivains, les intellectuels fournissent un récit, une narration de l'enracinement dans un pays originel, voire d'un ressourcement symbolique qui reconstruit une «imagined community» (Anderson, 1983) largement indépendante des appareils institutionnels à l'œuvre dans le cas des États-nations. 


\section{Quelle présence féminine?}

La présence des femmes dans les recherches sur le transnationalisme demeure excessivement limitée (Pessar et Mahler, 2003). Pourtant, les mouvements migratoires ont connu une féminisation certaine et l'on ne peut pas dire que les femmes migrantes ne mènent pas une vie qui traverse les frontières. Dahinden (2005), par exemple, met en lumière la participation des femmes albanaises en Suisse à la «culture transnationale », notamment par le biais de la renégociation des rapports de genre à l'intérieur du couple et de la famille tant dans le pays d'immigration qu'en cas de retour au pays d'origine. Il semble bien que l'invisibilité des femmes dans les études du transnationalisme tienne, entre autres, au fait que ces liens sont thématisés sous le terme de famille transnationale (Bryceson et Vuorela, 2002 ; Evergeti et Zontini, 2006) et ainsi en quelque sorte refoulés dans le domaine privé.

Il existe certes des rares études de cas intéressantes, qui analysent les migrations des femmes et notamment leurs activités commerciales et professionnelles dans l'optique du transnationalisme (Al-Sharmani, 2006 ; Schmoll, 2005) ; les études de terrain présentées dans ce dossier sont de celles-là. Elles font état d'une présence des femmes, sans doute minoritaire parmi les acteurs transnationaux eux-mêmes minoritaires, qui, n'est pas cantonnée à la seule sphère familiale et investit la sphère publique. Ainsi relève-t-on le rôle central des femmes dans la production musicale d'une world music aux racines capverdiennes ou encore celui des femmes chinoises dans les activités économiques. Les femmes investissent en outre le champ plus proprement politique, tant dans les activités tournées vers le pays d'origine (c'est le cas par exemple des Sénégalaises) que, de manière très profilée et professionnelle, dans les activités politiques ancrées dans le pays de résidence, comme le démontre le cas des femmes turques de la deuxième génération en Suisse.

\section{Quelle intégration?}

S'il y a une question qui traverse en filigrane la littérature européenne sur le phénomène du transnationalisme, c'est bien celle de l'éventuelle contradiction entre les activités transnationales et l'intégration des immigrés dans la société de résidence. Deux points de vue existent sur cette question : le premier considère le transnationalisme comme opposé à l'assimilation, comme un changement de paradigme, alors que selon le second, ces deux options sont compatibles. La signification traditionnelle de l'assimilation comporte l'idée d'absorption et possède une connotation normative (Brubaker, 2001) ; à cet égard, le maintien des liens sociaux avec le pays d'origine est envisagé comme une menace pour le résultat attendu et apparaît comme profondément contradictoire. D'autres experts contestent cette opposition binaire entre l'assimilation et les liens transnationaux. L'École de Chicago interprète ces pratiques comme une étape transitoire traversée par certains groupes dans leur processus d'assimilation, en les situant dans un cadre politique d'États-nations distincts, une perspective qui résout la contradiction entre le «transnationalisme » (ante litteram) et l'assimilation. 
En Europe, l'opposition entre les activités transnationales et l'assimilation a, jusqu'à maintenant, été plus thématisée dans un cadre normatif en référence à une conception de l'intégration comme un jeu à somme nulle; ce dossier l'aborde en revanche comme une question empirique. Nombre de contributions, chacune à leur manière, discute la crainte d'antagonisme entre la formation et le maintien de liens forts entre individus d'un groupe et la formation de liens entre individus appartenant à divers groupes.

Toutes les études de terrain de ce dossier cependant aboutissent à la conclusion qu'il ne saurait y avoir de contradiction entre investissement dans les activités transnationales et intégration dans le pays d'immigration parmi les groupes étudiés. Il n'y a pas d'antagonisme entre «bonding» et «bridging»(Putnam, 2000), puisque l'intégration ne comporte pas la perte de l'identité culturelle propre, mais une implication plus forte dans un autre univers culturel et social. D'ailleurs, l'absence de liens ne semble guère un indicateur de plus grande intégration, comme le démontrent les cas des Portugais en Suisse, des Marocains et des Roumains en Italie, De plus, nous l'avons vu, faute d'être sortis de la précarité, les groupes immigrés — du moins ceux étudiés dans notre projet — ne se mobilisent guère sur le plan transnational.

À l'instar de Lu et Touali (2007: 41) au terme de leur travail de terrain en Belgique, nous pouvons conclure, pour l'ensemble des études menées, que la relation entre activités transnationales et intégration s'articule de diverses manières. Pour entreprendre ces activités, les migrants doivent être déjà fonctionnellement intégrés dans la société d'accueil ; toutefois leur relative insatisfaction quant à leur situation - qu'elle soit choisie ou imposée - les pousse à s'investir dans des activités transnationales. Dans ce contexte, la mobilisation des ressources nécessaires à mener à bien leurs activités transnationales augmente la capacité des individus à s'insérer dans la société de résidence et de cette interaction naît une féconde transformation réciproque des représentations des groupes activement impliqués, migrants et autochtones.

\section{CONCLUSIONS}

L'objectif poursuivi par ce projet était celui de fournir une réponse à la question de savoir si l'Europe, avec ses nombreux États-nations et ses flux migratoires importants, connaissait des formes de transnationalisme comparables à celles observées dans l'espace anglo-saxon. Nos enquêtes de terrain indiquent clairement que tel est le cas, notamment si l'on prend soin d'élargir le champ d'observation au transnationalisme « grassroots », évitant de restreindre l'observation aux seules activités politiques, une tendance bien enracinée en Europe continentale. Reste que, dans les deux espaces, les activités transfrontalières sont le fait d'une minorité à l'intérieur des groupes immigrés des deux côtés de l'Atlantique.

Mais les observations mettent en lumière un paradoxe : d'une part, les activités transnationales sont le fait des individus les mieux dotés en ressources sociales, culturelles et économiques à l'intérieur de chaque groupe, ceux qui participent le mieux à la vie sociale du pays de résidence, qu'ils soient entrepreneurs, réfugiés, 
intellectuels, une minorité parmi les migrants. D'autre part, certains groupes seulement s'investissent dans des activités transfrontalières. Par ailleurs on ne peut identifier un pays - qu'il soit d'origine ou d'immigration — que l'on puisse associer à une forme plus développée de transnationalisme dans tous les cas de figures. La présence ou l'absence d'activités transnationales est ainsi le résultat de l'interaction historiquement située entre condition spécifique du groupe immigré et société d'immigration.

Les conditions à réunir pour qu'il y ait mobilisation transnationale semblent être : un groupe immigré qui soit, d'une part, suffisamment varié dans sa composition et doté de ce fait de ressources sociales, culturelles et économiques multiples et qui, d'autre part, vit dans une condition d'infériorisation dans la société d'immigration. Les activités transnationales constituent dès lors autant de réactions à des situations d'impasse que des manières d'accumuler du prestige social et culturel ou du pouvoir économique afin de négocier des meilleures conditions d'insertion aussi bien pour les individus que pour les groupes dont ils sont issus, voire pour atteindre des buts politiques. Dans ces conditions, les pratiques transnationales apparaissent comme une stratégie de «boundary blurring » (Bauböck, 1994a ; Zolberg et Long, 1999) : un brouillage des frontières qui implique la superposition d'identités collectives jusque là considérées comme séparées et mutuellement exclusives et qui comporte l'apprivoisement, la domestication de ce qui était considéré comme des différences « étrangères ».

\section{Références bibliographiques}

AL-SHARMANI Mulki (2006) "Living transnationally: Somali diasporic women in Cairo." International migration, 44(1): 55-77.

ALBA Richard et VICTOR Nee (2003) Remaking the American Mainstream: Assimilation and Contemporary Immigration. Cambridge: Harvard University Press.

ANDERSON Benedict (1983) Imagined Communities: Reflections on the Origin and Spread of Nationalism. London.

BAUBÖCK Rainer (1994a) The integration of immigrants. Strasbourg: Council of Europe.

BAUBÖCK Rainer (1994b) Transnational Citizenship: Membership and Rights in International Migration. Aldershot: Elgar.

BAUBÖCK Rainer (2003) "Towards a Political Theory of Migrant Transnationalism." International Migration Review, 37(3): 700-723.

BERTHOMIÈRE William et HILY Marie-Antoinette (2006) «Décrire les migrations internationales : les expériences de la co-présence ». Revue européenne des migrations internationales, 22(2) : 67-82.

BOLZMAN Claudio (2002) "De l'exil à la diaspora. L'exemple de la migration chilienne", in Fibbi, Rosita et Jean-Baptiste Meyer (éd.), Diasporas, développements, mondialisations. Paris : Autrepart. $\mathrm{N}^{\circ}$ spécial.

BRUBAKER Rogers (2001) "The Return of Assimilation? Changing Perspectives on Immigration and Its Sequels in France, Germany, and the United States." Ethnic and Racial Studies, 24(4): 531-548.

BRYCESON Deborah F. et VUORELA Ulla (éd.) (2002) The Transnational Family: New European Frontiers and Global Networks. Oxford: Berg. 
COHEN Robin (1997) "Caribbean Peoples as A Cultural Diaspora", in Cohen, Robin (éd.), Global Diasporas. London: UCL Press.

D'AMATO Gianni, OEGELMAN Nedim et SANTEL Bernhard (1997) Manna from the Bottom? Immigrant Strategies and Discourse Coalitions in Germany, Switzerland and the US. GAAC Meeting. Berlin.

DAHINDEN Janine (2005) "Contesting transnationalism? Lessons from the study of Albanian migration networks from former Yugoslavia." Global Networks, 5(2): 3-20.

EVERGETI Venetia et ZONTINI Elisabetta (2006) "Some critical reflections on social capital, migration and transnational families." Ethnic and Racial Studies, 29(6): 1025-1039

FAIST Thomas (2000) "Transnationalization in International Migration: Implications for the Study of Citizenship and Culture." Ethnic and Racial Studies, 23(2): 189-222.

FIBBI Rosita (1997) Les différentes modalités de vote des expatriés dans leur pays d'origine. Conférence sur les liens entre les Européens vivant à l'étranger et leur pays d'origine. Paris, 10-11 juin 1997 : Assemblée parlementaire du Conseil de l'Europe : 16.

FIBBI Rosita et MEYER Jean-Baptiste (2002) «Introduction : le lien plus que l'essence » Autrepart Numéro thématique : Diasporas, développements et mondialisations : 5-21.

FISHMAN Joshua Aaron (1964) "Language maintenance and language shift as a field of inquiry. A definition of the field and suggestions for its further development." Linguistics, 9: 32-70.

FOURON George et GLICK SCHILLER Nina (2002) "The Generation of Identity: Redefining the Second Generation Within a Transnational Social Field", in Levitt, Peggy et Mary Waters (éd.), The Changing Face of Home. The Transnational Lifes of Second Generation. New York: Russel Sage, p. 168-208.

GLICK SCHILLER Nina, BASCH Linda et SZANTON BLANC Cristina (1992). "Transnationalism: A New Analytic Framework for Understanding Migration." Annals of the New York Academy of Science, 645(1-24).

GORDON Milton M. (1964) Assimilation in American Life. The Role of Race, Religion and National Origins. New York: Oxford University Press.

HAMMAR Thomas (1990) Democracy and the Nation-State. Aliens, Denizens and Citizens in a World of International Migration. Aldershot: Avebury.

KIVISTO Peter (2001). "Theorising transnational immigration: a critical review of current efforts." Ethnic and Racial Studies, 24(4): 549-577.

KOOPMANS Ruud et STATHAM Paul (2001) "How national citizienship shapes Transnationalism. A Comparative Analysis of Migrant Claims-making in Germany, Great Britain and the Netherlands." Revue Européenne des Migrations Internationales, 17(2) : 63100.

LA BARBA Morena et CATTACIN Sandro (2007) Les associations italiennes en Suisse. Suisse : Controtempo.

LEVITT Peggy (2002) "The Ties That Change: Relations to the Ancestral Home over the Life Cycle", in Levitt, Peggy et Mary Waters (éd.), The Changing Face of Home. The Transnational Lifes of Second Generation. New York: Russel Sage, p. 123-144.

LOGAN John R., ALBA Richard D. et L. MCNULTY Thomas (2003) "Ethnic economies in metropolitan regions: Miami and beyond." Social Forces, 72(3): 691-724.

LU Haina et TOUALI Merouane (2007) Transnational practices of Immigrants in Western Europe. The case of Chinese and Maroccans in Belgium. Louvain-La-Neuve.

LUCASSEN Jan et LUCASSEN Leo (éd.) (1997) Migration, Migration History, History: Old Paradigms and New Perspectives. Bern: Peter Lang.

LUCASSEN Leo (2002) "Old and New Migrants in the Twentieth Century: A European Perspective." Journal of American Ethnic History, summer 2002: 85-101.

MA MUNG Emmanuel (1992) «Dispositif économique et ressources spatiales : Éléments d'une économie de diaspora ». Revue Européenne des Migrations Internationales, 8(3) : 175-194. 
NAUCK Bernhard, KOHLMANN Annette et DIEFENBACH Heike (1997) "Familiäre Netzwerke, intergenerative Transmission und Assimilationsprozesse bei türkischen Migratenfamilien." Kölner Zeitschrift für Soziologie und Sozialpsychologie, 49(3): 477-499.

ØSTERGAARD-NIELSEN Eva (2003) "The Politics of Migrants' Transnational Political Practices." International Migration Review, 37(760-786).

PACHE Véronique (1990) «Les associations portugaises de Suisse ou L'image d'une Suisse méconnue », in Gonseth, Marc-Olivier (éd.), Images de la Suisse = Schauplatz Schweiz. Berne : Société suisse d'ethnologie, pp. 339-354.

PESSAR Patricia et J. MAHLER Sarah (2003) "Transnational Migration: Bringing Gender In." International Migration Review, 37(3): 812-846.

PORTES Alejandro (1997) Globalization from Below: The Rise of Transnational Communities: Princeton, Princeton University.

PORTES Alejandro (1999) "Conclusion: Towards a new world - the origin and effects of transnational activities." Ethnic and Racial Studies, 22(2): 463-477.

PORTES Alejandro (2001) "New Research and Theory on Immigrant Transnationalism." Global Networks, 1(3, special issue).

PORTES Alejandro, GUARNIZO Luis E. et LANDOLT Patricia (1999) "Introduction. The Study of Transnationalism: pitfalls and promise of an emergent research field." Ethnic and Racial Studies, 22(2): 217-37.

PRIES Ludger (éd.) (2001) New Transnational Social Spaces. International Migration and Transnational Companies. London: Routledge.

PUTNAM Robert D. (2000) Bowling Alone. The Collapse and Revival of Civic America. New York: Simon \& Schuster.

RAGAZZI Francesco (2008) The concept of 'diaspora' and the 'transnational social space'. Diaspora and Transnationalism Conceptual, Theoretical and Methodological Challenges. Florence, April 10-11, 2008.

RAIJMAN R. et TIENDA M. (2000) "Immigrants pathways to business ownership. A comparative ethnic perspective." International Migration Review, 34(3): 682-706.

ROGERS A. (2000) "A European Space for Transnationalism." Working paper at the Transnational Communities Programm, WPTC 2K-07.

RUMBAUT Rubén G. (2002) "Severed or Sustained Attachments? Language, Identity and Imagined Communities in the Post-Immigrant Generation", in Levitt, Peggy et Mary Waters (éd.), The Changing Face of Home. New York: Russel Sage Foundation, p. 43-95.

SCHMOLL Camille (2005) «Pratiques spatiales transnationales et stratégies de mobilité de commerçantes tunisiennes ». Revue Européenne des Migrations Internationales, 21(1) : 131154.

VERTOVEC Steven (2003) Migrant Transnationalism and Modes of Transformation. Conceptial and Methodological Developments in the Study of International Migration. Princeton, Princeton University.

WALDINGER Roger (2006) " "Transnationalisme" des immigrants et présence du passé ». Revue Européenne des Migrations Internationales, 22(2) : 23-41.

WATERBURY Myra A. (2008) Bridging the Divide: Towards a comparative framework for understanding kin-state and migrant sending-state diaspora politics. Diaspora and Transnationalism Conceptual, Theoretical and Methodological Challenges. Florence, April 10-11, 2008.

WIMMER Andreas (2004) "Does Ethnicity Matter? Everyday Group Formation in Three Swiss Immigrant Neighborhoods." Ethnic and Racial Studies, 27(1): 1-36.

WIMMER Andreas et Glick Schiller Nina (2002) "Methodological nationalism and beyond : nation-state building, migration and the social sciences." Global Networks, 2(4): 301-334.

ZOLBERG Aristide et Long Litt Woon (1999) "Why Islam is like Spanish: Cultural Incorporation in Europe and the United States." Politics and Society, 27: 5-38. 